\title{
Note on rational approximations of the exponential function at rational points
}

\section{Alain Durand}

Let $p, q, u$, and $v$ be any four positive integers, and let $\delta$ be a number in the interval $0<\delta \leq 2$. In one of his papers, Kurt Mahler, Bulz. Austral. Math. Soc. 10 (1974), 325-335, proved that if $q$ satisfies the inequalities

$$
\begin{gathered}
q \geq e^{\{64(u+v)\}^{10 / \delta}}, \quad q \geq e^{8 u / \delta v}, \\
q \geq e^{\left(e^{2} u / v\right)^{24 / \delta}}, \text { and } q \geq e^{e^{(e \delta)^{-2}}},
\end{gathered}
$$

then

$$
\left|e^{u / v}-\frac{p}{q}\right|>q^{-(2+\delta)}
$$

In this note, by a slightly different treatment of some inequalities in Mahler's paper, we easily obtain the same result with $q$ only restricted by the first condition.

1 .

Denote by $n, v$, two positive integers and put

$$
P_{1}(x)=\sum_{k=n-1}^{2 n-1} \frac{n k ! v^{k-n+1}}{(k-n+1) !(2 n-k-1) !}(-x)^{2 n-k-1}
$$

Received 3 March 1976. 
and

$$
P_{2}(x)=\sum_{k=n}^{2 n-1} \frac{k ! v^{k-n+1}}{(k-n) !(2 n-k-1) !}(-x)^{2 n-k-1}
$$

Furthermore put

$$
f_{1}(x, z)=\frac{x^{n-1}}{(n-1) !}(v x-z)^{n} \text { and } f_{2}(x, z)=\frac{v x^{n}}{(n-1) !}(v x-z)^{n-1} \text {. }
$$

It follows from the definition of $P_{1}(x), P_{2}(x)$ that

$$
\left.\sum_{k \geq 0} \frac{\partial^{k}}{\partial x^{k}} f_{i}(x, z)\right|_{x=0}=P_{i}(z) \quad(i=1,2)
$$

and

$$
\left.\sum_{k \geq 0} \frac{\partial^{k}}{\partial x^{k}} f_{1}(x, z)\right|_{x=z / v}=P_{2}(-z),\left.\sum_{k \geq 0} \frac{\partial^{k}}{\partial x^{k}} f_{2}(x, z)\right|_{x=z / v}=P_{1}(-z) .
$$

Then, by Hermite's identity,

$$
\begin{aligned}
& e^{z / v_{P_{1}}(z)-P_{2}(-z)=e^{z / v}} \int_{0}^{z / v} e^{-t} f_{1}(t, z) d t= \\
& =z^{2 n} e^{z / v} \int_{0}^{1 / v} \frac{x^{n-1}}{(n-1) !}(v x-1)^{n} e^{-z x} d x \text {, }
\end{aligned}
$$

and

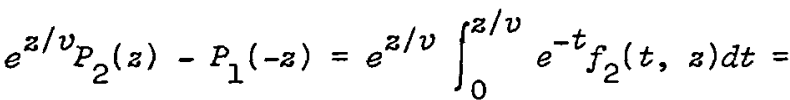

$$
\begin{aligned}
& =z^{2 n} e^{z / v} \int_{0}^{1 / v} \frac{x^{n}}{(n-1) !}(v x-1)^{n-1} e^{-z x} d x .
\end{aligned}
$$

Therefore, the determinant

$$
\Delta(z)=P_{1}(z) P_{1}(-z)-P_{2}(z) P_{2}(-z)
$$

is a polynomial in $z$ of the exact degree $2 n$ which has at $z=0$ a zero of order $2 n$. Then

$$
\Delta(z) \neq 0 \text { if } z \neq 0 \text {. }
$$


2 .

Denote by $p, q, u$, and $v$ four positive integers, and let

$$
\theta=\left|q e^{u / v}-p\right|
$$

By putting $z=u$ in the preceding formulae, we obtain

$$
\left|q P_{2}(-u)-p P_{1}(u)\right| \leq \theta\left|P_{1}(u)\right|+q\left(e^{u / v}-1\right) \sup _{0 \leq t \leq u / v}\left|f_{1}(t, u)\right|
$$

and

$$
\left|q P_{1}(-u)-p P_{2}(u)\right| \leq \theta\left|P_{2}(u)\right|+q\left(e^{u / v}-1\right) \sup _{0 \leq t \leq u / v}\left|f_{2}(t, u)\right|
$$

Since

$$
P_{1}(u) P_{1}(-u)-P_{2}(u) P_{2}(-u) \neq 0 \text {, }
$$

at least one of the integers

$$
q P_{2}(-u)-p P_{1}(u) \text { and } q P_{1}(-u)-p P_{2}(u)
$$

is distinct from zero. It follows that

$$
1 \leq \theta\left|P_{i}(u)\right|+q e^{u / v} \sup _{0 \leq t \leq u / v}\left|f_{i}(t, u)\right|
$$

where $i=1$ or $i=2$.

We have

$$
\left|P_{1}(u)\right| \leq \sum_{k=n-1}^{2 n-1} \frac{n k ! v^{k-n+1} u^{2 n-k-1}}{(k-n+1) !(2 n-k-1) !}=\sum_{j=0}^{n} \frac{n(n+j-1) !}{j !(n-j) !} v^{j} u^{n-j} \leq \frac{(2 n-1) !}{(n-1) !}(u+v)^{n}
$$

and

$$
\left|P_{2}(u)\right| \leq v \sum_{j=0}^{n-1} \frac{(n+j) ! v^{j} u^{n-1-j}}{j !(n-1-j) !} \leq v \frac{(2 n-1) !}{(n-1) !}(u+v)^{n-1}
$$

Next, when $t$ lies in the interval $0 \leq t \leq u / v$,

$$
\begin{aligned}
\max \left\{\left|f_{1}(t, u)\right|,\left|f_{2}(t, u)\right|\right\} \leq \frac{u^{2 n-1}}{(n-1) ! v^{n-1}} \sup _{0 \leq t \leq 1}(t(1-t))^{n-1} & \leq \\
& \leq \frac{u^{2 n-1}}{(n-1) !(4 v)^{n-1}} \leq \frac{u^{2 n-1}}{(n-1) ! 4^{n-1}} .
\end{aligned}
$$


Then, by (1), we can write

$$
1 \leq \frac{(2 n-1) !}{(n-1) !}(u+v)^{n} \theta+q \frac{e^{u / v} u^{2 n-1}}{(n-1) ! 4^{n-1}} .
$$

3.

Denote by $m_{0}$ the smallest integer which satisfies

$$
2 q e^{u^{2 m_{0}+1}} \leq m_{0} ! 4^{m_{0}}
$$

From the definition of $m_{0}$, it follows that

$$
\left(m_{0}-1\right) ! 4^{m_{0}-1}<2 q e^{u^{2 m_{0}-1}}
$$

Since

$$
\frac{\left(2 m_{0}+1\right) !}{m_{0} !}=\left(\begin{array}{c}
2 m_{0}+1 \\
m_{0}
\end{array}\right) \cdot\left(m_{0}+1\right) !<2^{2 m_{0}+1}\left(m_{0}+1\right) !,
$$

we have, by (2), (3), and (4), with $n=m_{0}+1$,

$$
1 \leq 2.2^{2 m_{0}+1}(u+v) m^{m_{0}+1} \theta .2 q e^{u^{2 m_{0}-1}} m_{0}\left(m_{0}+1\right) 4^{1-m_{0}} \text {. }
$$

Note that

$$
m_{0}\left(m_{0}+1\right) \leq 2^{2 m_{0}-1}, u+v>u \text {, and } m_{0} \geq 1
$$

then

$$
I \leq \theta q e^{u}(4(u+v))^{3 m_{0}}
$$

4.

Now, we require an upper estimate for $m_{0}$. By (4), we have

$$
m_{0} ! 4^{m_{0}-1}<2 q e^{u_{u}^{2 m_{0}-1}} m_{0}
$$

Since 


$$
m_{0} ! \geq m_{0}^{m_{0}+\frac{1}{2}} e^{-m_{0}} \sqrt{2 \pi}, \quad m_{0}^{\frac{1}{2}} \leq \frac{2^{m_{0} / 2}}{\sqrt{2}} \text {, and } \frac{\sqrt{2} e}{4}<1 \text {, }
$$

it follows that

$$
m_{0}^{m_{0}}<q e^{u^{2 m_{0}-1}} \cdot \frac{2 e^{m_{0} m_{0}^{\frac{2}{2}}}}{4^{m_{0}-1} \sqrt{2 \pi}} \leq q e^{u^{2 m_{0}}} u^{\frac{4}{\sqrt{\pi}}}\left(\frac{\sqrt{2} e}{4}\right)^{m_{0}}<3 q e^{u^{2 m_{0}}} .
$$

Put

$$
b=\frac{1}{u^{2}} \log \left(3 q e^{u}\right) \text { and } x=\frac{m_{0}}{u^{2}}
$$

Hence

$$
x^{x}<e^{b}
$$

Suppose that

$$
b \geq 27>e^{e} .
$$

Then, the condition

$$
x \geq \frac{b}{\log (b / \log b)}
$$

implies

$$
x \log x \geq b+\frac{b \log \log \log b}{\log (b / \log b)} \geq b .
$$

Hence, by $(7)$,

$$
x<\frac{b}{\log (b / \log b)} \text { and } m_{0}<\frac{b u^{2}}{\log (b / \log b)} \text {, }
$$

provided $b \geq 27$.

$$
5 .
$$

We can now prove the

THEOREM. Let $\delta$ be a constant in the interval $0<\delta \leq 2$, and let $p, q, u$, and $v$ be four positive integers. Assume that

$$
q \geq e^{(4(u+v))^{10 / \delta}}
$$


Then

$$
\left|e^{u / v}-\frac{p}{q}\right| \geq q^{-(2+\delta)}
$$

Proof. We have

$$
\log . q \geq(4(u+v))^{10 / \delta}>u^{10 / \delta} \geq u^{5} .
$$

Then

$$
b=\frac{1}{u^{2}} \log \left(3 q e^{u}\right)>\frac{\log q}{u^{2}} \geq(\log q)^{3 / 5} .
$$

Since

$$
5 e^{y / 5} \geq 3 y \text { for } y \geq 5 \log 5 \text {, and } \log q \geq 8^{10 / \delta} \geq 8^{5},
$$

it follows that

$$
\frac{b}{\log b}>\frac{5}{3} \frac{(\log q)^{3 / 5}}{\log \log q} \geq(\log q)^{2 / 5} \geq(4(u+v))^{4 / \delta} .
$$

Since

$$
b>(\log q)^{3 / 5} \geq 8^{3}>27 \text {, }
$$

we deduce from ( 8 ),

$$
3 m_{0} \log (4(u+v))<\frac{3 b u^{2} \log (4(u+v))}{\log (b / \log b)} \leq \frac{3 \delta}{4} \log \left(3 q e^{u}\right) .
$$

On substituting this upper estimate in (5), it follows that

$$
1<\theta q e^{u}\left(3 q e^{u}\right)^{3 \delta / 4} \text {. }
$$

Finally, since

$$
e^{u}\left(3 e^{u}\right)^{3 \delta / 4} \leq e^{6 u} \leq e^{(\delta / 4)(4 u)^{10 / \delta}} \leq q^{\delta / 4}
$$

we find that

$$
\theta \geq q^{-1-\delta}
$$

This completes the proof.

6.

Note that the condition for $q$ in this theorem can be easily replaced 
by a weaker one, for example if we suppose $0<\delta \leq 1$.

Compare also related results by Bundschuh [1].

\section{References}

[1] Peter Bundschuh, "Irrationalitätsmaße für $e^{a}, a \neq 0$ rational oder Liouville-Zahl", Math. Ann. 192 (1971), 229-242.

[2] Kurt Mahler, "On rational approximations of the exponential function at rational points", Bull. Austral. Math. Soc. 10 (1974), 325-335.

Département de Mathématiques,

U.E.R. des Sciences de Limoges,

Limoges,

France. 